\title{
La Raison dépossédée : Intentionnalité et liberté face à l'Autre chez Lévinas et Sartre
}

\author{
Par GIORGIA VASARI \\ Università degli Studi di Roma « La Sapienza »
}

À la lumière des déplorables événements qui ont marqué l'histoire occidentale, de nombreux intellectuels des années 1930 se sont interrogés sur la rationalité de l'histoire, la souveraineté de la Raison et le rôle de la liberté humaine. C'est dans ce contexte intellectuel que, prolongeant une réflexion inaugurée vingt ans plus tôt, Emmanuel Lévinas remarque en 1947 une « divergence entre les événements et l'ordre rationnel ${ }^{1}$, tout en dénonçant « l'inaptitude de l'intelligence à ce qui devait en être la fonction essentielle $»^{2}$. À la même période, Jean-Paul Sartre s'explique à propos d'un nouveau sens de l'humanisme fondé sur la liberté humaine ${ }^{3}$. Cette liberté est le revers du fait incontournable de la contingence du réel - de son absence de rationalité - qui nourrit la pensée sartrienne dès ses tous débuts, dans les années 1920, lorsque son auteur constate l'impossibilité de rencontrer la nécessité dans le monde 4 .

Je propose de comparer certains aspects des philosophies de Lévinas et de Sartre sur ce thème, à savoir, le rapport entre Raison et liberté. Le choix de rapprocher ces deux penseurs malgré leurs différences est motivé, d'une part, par leur commune appartenance au mouvement phénoménologique, et, d'autre

\footnotetext{
${ }^{1}$ E. Lévinas, De l'existence à l'existant, Paris, Vrin, 1990, p. 23 (désormais cité EE). ${ }^{2}$ Ibid.

${ }^{3}$ J.-P. Sartre, L'Existentialisme est un humanisme, Paris, Nagel, 1952, passim.

${ }^{4}$ J.-P. Sartre, Empédocle dans A. Chabot [dir.], Études sartriennes, vol. 20 : Inédits de jeunesse, Paris, Garnier, 2016, p. 38 sq ; S. de Beauvoir, La Cérémonie des adieux, Paris, Gallimard, 1987, p. 199-200 ; V. de Coorebyter, Sartre avant la phénoménologie, Bruxelles, Ousia, 2005, p. 17-62.
} 
part, plus spécifiquement, par l'attention que l'un et l'autre accordent à l'intentionnalité husserlienne ${ }^{1}$. En effet, je retiens que la manière dont Lévinas et Sartre s'approprient cette notion clef permet de comprendre les similitudes et les différences entre leurs philosophies respectives. Bien plus, elle enveloppe une nouvelle conception des rapports entre Raison et liberté.

J'aborderai la question en deux temps. D'une part, je soutiendrai que Sartre et Lévinas opèrent non seulement de concert une analyse de la souveraineté de la Raison qui s'attaque principalement à l'idéalisme dans son acception la plus large, mais exploitent aussi, au sein de la phénoménologie qu'ils réélaborent tout en la découvrant, le concept husserlien d'intentionnalité, qui doit théoriser la possibilité d'une « sortie » - d'une « évasion $»^{2}$ ou d'un « désengluement $»^{3}$ — de l'être. D'autre part, j'exposerai les appropriations de l'intentionnalité qui furent réalisées par Lévinas et Sartre. J'examinerai ainsi la manière dont s'articulent chez eux les concepts de Raison et de liberté. Cette articulation sera examinée sous deux points de vue différents. J'analyserai d'abord la forme qu'elle prend au sein des relations qui unissent la conscience et le monde. Ensuite, j'indiquerai comment elle évolue à l'intérieur d'un autre contexte : celui des relations intersubjectives qui unissent deux consciences.

Sartre et Lévinas accordent un rôle majeur à la liberté : celle-ci doit détrôner la Raison. Or, pour démontrer que ces auteurs chargent ce concept du même enjeu, je m'intéresserai plus spécifiquement à deux de leurs développements. J'examinerai leur critique commune du concept de Raison, avant de m'intéresser à l'usage qu'ils font du concept husserlien d'intentionnalité. Je monterai en effet que ces opérations établissent une séparation entre la liberté de la conscience et la Raison conçue comme « pouvoir de compréhension ». Mais, pour ce faire, je replacerai ces analyses dans un cadre plus global. Elles seront lues à la lumière de la problématique de l'intersubjectivité, c'està-dire dans le contexte d'une « rencontre » entre deux consciences. Bien évidemment, je ne soutiendrai pas que les positions de Lévinas et de Sartre convergent d'un point de vue théorique ; je soutiendrai plutôt que l'intentionnalité permet, en tant que fil conducteur de lecture, non seulement de développer à

${ }^{1}$ Cf. E. Lévinas, La Conscience non intentionnelle dans Id., Entre nous : essais sur le penser-à-l'autre, Paris, Grasset, 1991, p. 141-151 ; C. Pagès, " "Distance et proximité" : les deux lectures lévinassiennes de l'intentionnalité », Bulletin d'analyse phénoménologique $\mathrm{n}^{\circ} 8$ (2010), p. 264-283 ; J.-P. Sartre, Une idée fondamentale de la phénoménologie de Husserl : l'intentionnalité, dans Id., La Transcendance de l'Ego et autres textes phénoménologiques, Paris, Vrin, 2003, p. 88-90 (désormais cité Intentionnalité).

${ }^{2}$ E. Lévinas, De l'évasion, Paris, Poche, 1998 (désormais cité $D E$ ).

${ }^{3}$ J.-P. Sartre, L'Être et le Néant, Paris, Gallimard, 1987, p. 58 sq. (désormais cité EN). 
propos de ces deux philosophies une réflexion rigoureuse sur les rapports entre Raison et liberté, mais rend surtout possible la compréhension des différences plus profondes qui subsistent entre elles. En raison des convergences de leurs analyses phénoménologiques de la nausée et de la honte, les pensées lévinassiennes et sartriennes ont souvent fait l'objet de lectures comparatives. Dans ces conditions, j'examinerai leurs premiers travaux ${ }^{1}$ et leurs magna opera ${ }^{2}$. C'est, en effet, dans ces textes que se concentrent leurs appropriations de l'intentionnalité, leurs critiques du primat de la raison théorique, et leurs dialogues mutuels autour du concept d'altérité . $^{2}$

\section{Lévinas : la Raison qui ne libère pas}

Dans l'un de ses premiers écrits, De l'évasion (1934), Lévinas reconnait qu'il fait partie d'une « génération $»^{4}$ qui condamne la « philosophie de l'être $»^{5}$, qui est la «marque d'une certaine civilisation, installée dans le fait accompli de l'être et incapable d'en sortir " ${ }^{6}$. Lévinas prône une « sortie $»^{7}$ de l'être et affirme derechef que le besoin d'une telle évasion est le «mal du siècle $\rangle^{8}$. Après avoir esquissé une synthèse de l'histoire de la pensée occidentale, il soutient que cette pensée culmine avec l'idéalisme avant d'échouer avec lui ${ }^{9}$ :

[L]e progrès n'a pas amené la philosophie occidentale à dépasser entièrement l'être. Lorsqu'elle découvrit au-delà des choses — modèle premier de l'être les domaines de l'idéal, de la conscience et du devenir, elle fut incapable de les priver d'existence, car tout le bénéfice de sa découverte consistait précisément

\footnotetext{
${ }^{1}$ Je me réfère en particulier à E. Lévinas, Théorie de l'intuition dans la phénoménologie de Husserl, Paris, Vrin, 2001 (désormais cité TIH) ; De l'évasion, op. cit. ; Le Temps et l'Autre, Paris, PUF, 1983 (désormais cité TA) ; De l'existence à l'existant, op. cit.; mais aussi à J.-P. Sartre, Une idée fondamentale de la phénoménologie de Husserl : l'intentionnalité, op. cit. ; La Transcendance de l'Ego, op. cit. ; La Nausée, Paris, Gallimard, 1972 (désormais cité $L N$ ).

${ }^{2}$ E. Lévinas, Totalité et Infini, Paris, Poche, 1990 (désormais cité TI) ; J.-P. Sartre, L'Être et le Néant, op. cit.

${ }^{3}$ Sur ce point, cf. G. Hanus, Echapper à la philosophie ? Lecture de Lévinas, Lagrasse, Verdier, 2012, p. 153-167.

${ }^{4} D E$, p. 94.

${ }^{5}$ Ibid.

${ }^{6}$ Ibid., p. 99.

${ }^{7}$ Ibid., p. 127.

${ }^{8}$ Ibid., p. 94.

${ }^{9}$ Ibid., p. 91-99, 124-127.
} 
à la leur attribuer. L'ontologisme sous sa signification la plus large restait le dogme fondamental de toute pensée [...]. D'ailleurs dans la pensée contemplative, la théorie est dans son fond le comportement de celui qui porte à jamais le stigmate de l'existence : elle est essentiellement soumise à l'existant et quand elle ne part pas de l'être elle va au-devant de lui. C'est l'impuissance devant le fait accompli. La connaissance est précisément ce qui reste à faire quand tout a été accompli. [...] L'affranchissement de l'idéalisme à l'égard de l'être est basé sur sa sous-estimation. Dès lors, au moment même où l'idéalisme s'imagine l'avoir dépassé il en est envahi de toutes parts. Ces relations intellectuelles dans lesquelles il a dissous l'univers n'en sont pas moins des existences — ni inertes, ni opaques certes - mais qui n'échappent pas aux lois de l'être. L'idéalisme n'est pas seulement exposé aux attaques de tous ceux qui lui reprochent de sacrifier la réalité sensible, de méconnaître et de mépriser les exigences concrètes et poignantes de l'homme en proie à ses problèmes quotidiens, d'être par conséquent incapable de diriger et de conduire ; il n'a même pas l'excuse d'échapper à l'être, car, sur le plan où il nous amène, il trouve sous une forme plus subtile et qui invite à une fausse sérénité l'être toujours le même qui n'a renoncé à aucun de ses caractères ${ }^{1}$.

Même si Lévinas admire la tentative idéaliste d'outrepasser la simple expérience sensible ${ }^{2}$, son argumentation s'attaque à la connaissance, à la théorie, à la pensée contemplative, impuissante et « soumise à l'existant $»^{3}$. Cette critique du primat de la théorie avait déjà été esquissée par Lévinas dans le cadre de sa thèse de troisième cycle. L'intellectualisme husserlien y est perçu comme une limite pour la phénoménologie, car il ne rend justice ni à l'existence concrète, ni à la vie dans son caractère " riche et multiforme ${ }^{4}$. Plus généralement, on peut dire que c'est tout l'idéal classique qui est fustigé par ce texte qui déclare qu' «Auschwitz a été commis par la civilisation de l'idéalisme transcendantal $»^{5}$. Le point spécifiquement visé par la critique lévinassienne concerne la logique de l'être qui est considérée, lato sensu, comme une dynamique

\footnotetext{
${ }^{1}$ Ibid., p. 124-127.

${ }^{2}$ Pour Lévinas, la compréhension idéaliste du réel renvoie à un « progrès » de la « philosophie occidentale » au sein duquel le « modèle de l'être » qu'offrent « les choses » est « dépassé » dans « les domaines de l'idéal, de la conscience et du devenir ». Toutefois, ce progrès, et la compréhension qui en découle, échoue ; car c'est dans « les relations intellectuelles » que l'idéalisme retrouve l'être qu'il croyait avoir dépassé. (cf. ibid., p. 124-127). Il semble bien que Lévinas s'attaque ici au cheminement de la conscience décrit par la Phénoménologie de l'esprit, Paris, Vrin, 2006, p. 131-144 ; 645662.

${ }^{3}$ DE, p. 125.

${ }^{4}$ TIH, p. 86.

${ }^{5}$ F. Poirié, Emmanuel Lévinas. Qui êtes-vous ?, Lyon, La Manufacture, 1987, p. 84.
} 
propre à la connaissance et à la souveraineté absolue du moi sur les choses. Cette mise en question apparaît en fait dès 1946 avec la formulation du concept d' $\left\langle\right.$ il y a $»^{1}$, qui est le revers de l'opération du paragraphe quarante neuf des Ideen $I^{2}$. Si la conscience transcendantale résiste à l'anéantissement du monde, parce qu'elle est la source absolue du sensé, elle surgit à un moment qui est, selon Lévinas, logiquement et ontologiquement second par rapport à l'«être en général $»^{3}$; par rapport à l' « il y a $\rangle^{4}$ qui « constitue le fond obscur de l'existence $»^{5}$ et qui fait $«$ horreur $»^{6}$ au sujet par son $«$ absurdité foncière $»^{7}$. Cette position du sujet au sein de l'être est une condamnation pour ce sujet même qui, « en participant à l'il y a, se retrouve comme solitude, comme le définitif de l'enchaînement d'un moi à son soi $\gg^{8}$. Toutefois, cette position ne relève pas exclusivement du tragique car la maîtrise de l'existence de la part de l'existant se configure aussi comme une « jouissance » et un «bonheur » au sein du monde, qui est considéré comme un ensemble de " nourritures $»^{9}$ pour l' « alimentation ${ }^{10}$ du sujet. Ce dernier est libre de jouir des existants et des choses du monde en les possédant. Lévinas explique en effet que le sujet — «l'hypostase $\|^{11}$ — « est liberté $»^{12}$, c'est à dire « maître de l'exister »; il « exerce sur son existence le viril pouvoir du sujet $»^{13}$. Donc, si, d'un côté, cette liberté

\footnotetext{
${ }^{1}$ E. Lévinas, « Il y a », Deucalion (1946/1), p. 141-154 ; EE, p. 81-91; TA, p. 25-30.

${ }^{2}$ E. Husserl, Idées directrices pour une phénoménologie et une philosophie phénoménologique pures, trad. fr. P. Ricœur, Paris, Gallimard, 1950, p. 160-164.

${ }^{3}$ EE, p. 81 .

${ }^{4}$ Ibid.

${ }^{5}$ Ibid., p. 84.

${ }^{6}$ Ibid., p. 83 sq.

${ }^{7}$ TA, p. 29.

${ }^{8}$ EE, p. 121.

${ }^{9}$ TA, p. 45 sq.

${ }^{10}$ Ibid. ; TI, p. 112 sq.

${ }^{11}$ Lévinas utilise ce terme technique pour désigner le sujet : « Nous cherchions l'apparition même du substantif. Et pour indiquer cette apparition nous avons repris le terme d'hypostase qui, dans l'histoire de la philosophie, désignait l'événement par lequel l'acte exprimé par un verbe devenait un être désigné par un substantif » (EE, p. 119-120). Lévinas se réfère au verbe « être » et désigne le sujet - l'hypostase comme l'étant (ou l'être) qui se pose au sein de l'être verbal et en devient le maître ; de sorte que son acte d'exister se configure comme un exercice de pouvoir.

${ }^{12}$ TA, p. 34.

${ }^{13}$ Ibid.
} 
caractérise le sujet qui est condamné à « l'être en général » dans son «absurdité foncière $»^{1}$, cette solitude n'est « pas seulement un désespoir et un abandon, mais aussi une virilité et une fierté et une souveraineté $»^{2}$.

La liberté caractérise la conscience qui maîtrise l'existence originairement anonyme et insensée. L'existant est indépendant et heureux s'il s'approprie librement les choses du monde. Cependant, cette liberté ne paraît pas tout à fait définitive. Elle semble mise en péril par la Raison qui exerce son pouvoir en répandant la « lumière » à travers laquelle se produit l'existence mondaine. Mais cette lumière qui pose l'autre, «le pose comme s'il sortait de moi » ${ }^{3}$ :

En englobant le tout dans son universalité, la raison se retrouve elle-même dans la solitude. Le solipsisme n'est ni une aberration ni un sophisme : c'est la structure même de la raison. Non point en raison du caractère « subjectif » des sensations qu'elle combine, mais en raison de l'universalité de la connaissance, c'est-à-dire de l'illimité de la lumière et de l'impossibilité pour aucune chose d'être en dehors. Par là la raison ne trouve jamais d'autre raison à qui parler. L'intentionnalité de la conscience permet de distinguer le moi des choses, mais ne fait pas disparaître le solipsisme puisque son élément, la lumière, nous rend maître du monde extérieur, mais est incapable de nous y découvrir un pair. L'objectivité du savoir rationnel n'enlève rien au caractère solitaire de la raison. Le retournement possible de l'objectivité en subjectivité est le thème même de l'idéalisme qui est une philosophie de la raison. L'objectivité de la lumière, c'est la subjectivité elle-même ${ }^{4}$.

Il est possible de comprendre pourquoi la pensée occidentale et l'idéalisme n'arrivent pas à se délivrer de l'être. D'après Lévinas, il est dans la nature de la Raison théorique - toujours raison d'un moi — de tout reconduire à soi, en engendrant une totalité. Lévinas explique en effet que la «transcendance de l'espace est assurée si elle est fondée sur une transcendance sans retour au point de départ $»^{5}$. La question lévinassienne concerne donc la condition d'une telle transcendance, qui est capable de libérer définitivement le moi de son enchaînement à soi. Cette problématique est clairement posée dans Totalité et Infini, notamment dans la Préface à l'édition allemande de 1987, où Lévinas déclare :

${ }^{1}$ Cf. supra, notes $21 \mathrm{sq}$.

${ }^{2}$ Ibid., p. 35.

${ }^{3}$ Ibid.

${ }^{4}$ Ibid., p. 47-48.

${ }^{5}$ Ibid., p. 48-49. 
Ce livre conteste que la synthèse du savoir, la totalité de l'être embrassée par le moi transcendantal, la présence saisie dans la représentation et le concept et l'interrogation sur la sémantique de la forme verbale de l'être - stations inévitables de la Raison - soient les instances ultimes du sensé. Ramènent-elles ou mènent-elles à la capacité d'assurer l'accord d'un monde et de manifester ainsi la Raison jusqu'au bout? La raison jusqu'au bout ou la paix entre les hommes ${ }^{1}$.

La question enveloppe assurément une critique de la rationalité théorique. Pour Lévinas, on ne peut avoir d'autrui une connaissance purement théorique. Si elle fonctionne sur les objets, la maitrise du moi devient un échec une fois appliquée à l'autre. Pire, elle se transforme en une tentative de soumission. Aussi, la « raison jusqu' au bout » renvoie donc à cette interrogation : que doit être l'affranchissement du moi ? S'il doit rendre possible la «paix entre les hommes $»^{2}$, et éviter «la divergence entre les événements et l'ordre rationnel $»^{3}$, en quoi doit-il consister?

\section{Sartre : la liberté hors de la Raison}

Dans Une idée fondamentale de la phénoménologie de Husserl : l'intentionnalitét, Sartre s'attaque à la « philosophie alimentaire [...] selon laquelle connaître c'est manger $»^{5}$. Il s'en prend apparemment à la téléologie de la connaissance et à la capacité de la Raison d'envelopper une rationalité intrinsèque du monde et de l'histoire. Par la même occasion, il salue l'intentionnalité husserlienne - ipso facto, radicalement réinterprétée — d'avoir contrecarré cette «philosophie digestive $»^{6}$. En effet, grâce à l'intentionnalité husserlienne, Sartre déclare que connaître c'est «s'éclater vers »", «s'arracher à la moite intimité » pour se retrouver finalement dans le monde, un monde « indifférent, hostile et rétif $»^{8}$ dont une philosophie de la compréhension de l'intériorité ne

\footnotetext{
${ }^{1}$ TI, p. II.

${ }^{2}$ Ibid.

${ }^{3}$ EE, p. 23.

${ }^{4}$ V. de Coorebyter, Sartre face à la phénoménologie, Bruxelles, Ousia, 2001, p. $27-$ 28.

${ }^{5}$ Intentionnalité, p. 87.

${ }^{6}$ Ibid.

${ }^{7}$ Ibid, p. 88.

${ }^{8}$ Ibid.
} 
saurait rendre compte, car « la connaissance ne [peut], sans malhonnêteté, se comparer à la possession $»^{1}$.

Comme Lévinas, Sartre semble donc critiquer la conception de la Raison comme compréhension du réel, mais aussi revendiquer la concrétude de l'existence dans le monde, au-delà de la seule théorie ${ }^{2}$. Évidemment, de nettes différences subsistent entre ces philosophes ; mais le primat d'une Raison vouée à la compréhension du réel, primat de la logique idéaliste propre à l'Esprit, semble bien être ce qui est mis en cause par les deux philosophes. De même, tous deux contestent la souveraineté de l'Ego. Contre toutes les philosophies transcendantales qui situent l'origine ultime du sens dans un pôle égologique, ils inversent les données du problème de la significativité.

Sur ce dernier point, avec La Transcendance de l'Ego, Sartre achève son parricide vis-à-vis de Husserl et sa réception personnelle de la phénoménologie, en purifiant le champ transcendantal de toute structure égologique ${ }^{3}$. C'est en effet la radicalisation du principe husserlien d'intentionnalité qui le conduit à thématiser l'idée d'une conscience transcendantale qui n'est " rien » ${ }^{4}$ puisque tout est en dehors d'elle, mais qui est en même temps « tout » ${ }^{5}$ parce qu'elle est dans le monde. En se constituant à la surface des choses, cette conscience impersonnelle devient une « sphère d'existence absolue, c'est-à-dire de spontanéités pures $»^{6}$. Elle dévoile les objets sur le mode du « ne pas l'être ». Elle entretient donc une stricte corrélation avec l'être mais, en même temps, elle ne l'est pas. En ce sens, elle s'arrache à l'être et se constitue comme liberté

\footnotetext{
${ }^{1}$ Ibid.

${ }^{2}$ Ibid., p. 89.

3 J.-P. Sartre, La Transcendance de l'Ego, dans Id., La Transcendance de l'Ego et autres textes phénoménologiques, op. cit., p. 93-131.

${ }^{4}$ Ibid., p. 125.

${ }^{5}$ Ibid.

${ }^{6}$ Ibid., p. 126. Même si la dualité ontologique du pour-soi et de l'en-soi n'est pas thématisée explicitement dans La Transcendance de l'Ego, à la même époque, on trouve dans l'Imagination (Paris, PUF, 2012, p. 1) la première formulation de ce dualisme : « Ces différentes qualités [...] se donnent à mon regard comme des existences que je puis seulement constater et dont l'être ne dépend aucunement de mon caprice. Elles sont pour moi, elles ne sont pas moi. [...] Cette inertie du contenu sensible, [...] c'est l'existence en soi. [...] Cette forme inerte, qui est en deçà de toutes les spontanéités conscientes, que l'on doit observer, apprendre peu à peu, c'est ce que l'on appelle une chose. En aucun cas, ma conscience ne saurait être une chose, parce que sa façon d'être en soi est précisément un être pour soi ».
} 
pure et absolue ${ }^{1}$, par rapport à un monde qui est par principe inassimilable mais destiné à demeurer toujours « dehors $»^{2}$.

Ces éléments suffisent à faire apparaître un abîme entre les théories lévinasienne et sartrienne de l'intentionnalité, et, ainsi, une distance irréfragable entre leurs conceptions respectives du rapport au monde. En effet, tout en partageant des objectifs théoriques communs, elles sont quasiment opposées. Pour Lévinas, le sujet s'alimente des objets du monde et peut, sur la base de ce modèle d'appropriation, connaître le monde. Pour Sartre, l'intériorisation et l'assimilation de l'objet dans la conscience ne peuvent jamais se réaliser. En effet, si, pour traiter le sujet de la connaissance, L'Être et le Néant, utilise les mêmes métaphores que Lévinas (la jouissance, l'alimentation et l'assimilation) ${ }^{3}$, les conclusions sartriennes en diffèrent radicalement :

Ainsi y a-t-il un mouvement de dissolution qui va de l'objet au sujet connaissant. Le connu se transforme en moi, devient ma pensée et par là même accepte de recevoir son existence de moi seul. Mais ce mouvement de dissolution se fige du fait que le connu demeure à la même place, indéfiniment absorbé, mangé et indéfiniment intact, tout entier digéré et cependant tout entier dehors, indigeste comme un caillou ${ }^{4}$.

L'impossibilité pour l'objet d'être dirigé par la conscience semble jouer un rôle important au sein de la théorie de la contingence sartrienne, dont une « loi » est énoncée au cœur de La Nausée où Sartre déclare : «L'essentiel c’est

\footnotetext{
${ }^{1}$ C. Romano, « La liberté sartrienne ou le rêve d'Adam », Archives de Philosophie ${ }^{\circ} 3$ (2000), p. 476-477.

${ }^{2}$ Cf. Intentionnalité, p. 89. Dans sa belle introduction à l'intentionnalité et La transcendance de l'Ego, Vincent de Coorebyter explique comment Sartre a retourné les Ideen contre elles-mêmes. « [L]'ultime assise de ce renversement réside, écrit-il, dans un respect scrupuleux du clivage ontologique qui domine l'ensemble des Ideen, selon la thèse même de Husserl [...] au début du $\S 76 »$ (V. de Coorebyter, Introduction, dans J.-P. Sartre, La Transcendance de l'Ego et autres textes..., op. cit., p. 41-42). Nous avons vu que ce dualisme est l'une des clefs de l'Intentionnalité, où Sartre offre une réponse aux philosophies digestives : l'être-au-monde ne peut se former par alchimie interne car le monde est inassimilable à la conscience, qui relève d'un régime ontologique irréductiblement différent. Comme Sartre le signale dans L'Imagination, le principe d'intentionnalité lui-même est inséparable de cette distinction « sur laquelle Husserl revient sans se lasser » : la « distinction radicale entre la conscience et ce dont il y a conscience », l'objet de visée étant « par principe hors de la conscience, "transcendant" » (ibid.).

${ }^{3}$ Cf. EN, p. 638 sq.

${ }^{4}$ EN, p. 639.
} 
la contingence [...] l'existence n'est pas la nécessité » ${ }^{1}$. À l'impossibilité d'assimiler l'objet ${ }^{2} \mathrm{~s}^{\prime}$ ajoute donc celle de le connaître d'une manière permanente ${ }^{3}$, c'est-à-dire conforme à une essence qui serait impliquée par une succession de profils se donnant avec la manifestation d'un phénomène. Sartre conteste que la conscience puisse appréhender une essence qui serait la « loi manifeste qui préside à la succession [des] apparitions [d'un existant] ${ }^{4}$. Dans La Nausée, il déclare à ce sujet que « le monde des explications et des raisons n'est pas celui de l'existence »: « un cercle n'est pas absurde, il s'explique très bien par la rotation d'un segment de droite autour d'une de ses extrémités [...] [mais] un cercle n'existe pas » ${ }^{5}$. Plus loin, il ajoute qu' « exister c'est être là, simplement ; [car] les existants apparaissent, se laissent rencontrer, mais on ne peut jamais les déduire $»^{6}$. C'est donc l'idée de « raison de la série », l'idée d'essence, qui est mise en question. En ce sens, la possibilité de la connaissance comme compréhension du réel est le point qui semble être contesté. Au sein de passages décisifs du roman de 1938, Sartre caractérise en termes d' " idées abstraites » et d' « idées d'homme » les idées de « passage » à l'acte ${ }^{7}$ et de « vision $»^{8}$ qui renvoient à la théorie de l'intuition des essences. Il rejette l'idée husserlienne de remplissement de l'intention en proposant une théorie opposée selon laquelle la connaissance n'est pas orientée téléologiquement vers un idéal de " plénitude » ${ }^{9}$, car " tout est [déjà] plein ${ }^{10}$. Ceci, d'ailleurs, paraît tout à fait

${ }^{1} L N$, p. 187

${ }^{2}$ Cf. supra, note 5 .

${ }^{3} E N$, p. 235 : «La permanence est pure possibilité pour un "ceci" d'être conforme à son essence. [...] La fusion idéale de ce qui manque avec ce à quoi manque ce qui manque, comme totalité irréalisable, hante le pour-soi et le constitue dans son être même comme néant d'être. C'est, disions-nous, l'en-soi-pour-soi, ou la valeur ».

${ }^{4}$ Cf. EN, p. $12-13$.

${ }^{5} L N$, p. 184.

${ }^{6}$ Ibid., p. 187.

${ }^{7}$ Ibid., p. 188-189.

${ }^{8}$ Ibid., p. 186.

${ }^{9}$ Cf. E. Husserl, Recherches logiques, trad. fr. H. Élie et al., Paris, PUF, 2012, § 21, p. 97-99 : « [L]es intentions signitives sont en soi "vides" et "ont besoin de plénitude". [...] À la représentation signitive, prise en elle-même, il manque [...] toute espèce de plénitude ; c'est seulement la représentation intuitive qui la lui amène et la lui incorpore par identification. [...] [P]lus la représentation est "claire", plus sa "vivacité" est grande, plus le degré de figuration auquel elle est arrivée est élevé : plus elle est riche en plénitude. En conséquence, l'idéal de la plénitude serait atteint dans une représentation qui enfermerait dans son contenu phénoménologique son objet plein et entier ». ${ }^{10}$ Cf. LN, p. 188-189 : « Mes yeux ne rencontraient jamais que du plein. [...] Tout était plein, tout en acte $»$. 
cohérent avec la lecture qu'il propose de l'intentionnalité husserlienne dans sa note de 1934, où il indique que l'« on ne peut pas dissoudre les choses dans la conscience $»^{1}$.

Ces analyses nous permettent de mieux comprendre pourquoi «l'assimilation de la réalité » est un modèle qui est refusé par Sartre là où il est accepté par Lévinas ${ }^{2}$. Plus largement, elles nous permettent de qualifier plus précisément un aspect spécifique de leurs philosophies respectives : la manière dont elles conçoivent les relations existant entre les concepts de raison et de liberté. Pour Lévinas, le sujet émerge à partir d'un horizon absurde, quasiment inconcevable du point de vue de la conscience. En se posant dans l'existence, l'individu confère un sens au monde ; il le possède et s'en alimente librement et joyeusement. Toutefois, en raison de ces dynamiques d'appropriation intentionnelle et de dissolution de l'extériorité dans le moi, le sujet demeure " rivé » à lui-même, enchaîné à sa solitude, si bien que sa liberté n'est ni complète ni définitive ${ }^{3}$. Chez Sartre, la conscience surgit face à l'être en marquant une scission nette qui demeure à jamais irréconciliable. Pour cette raison, l'assimilation complète du monde au sein de la conscience n'est pas concevable et la conscience se configure comme une liberté absolue non substantielle ${ }^{4}$. Ainsi, elle sera toujours nécessairement transcendance de l'objet, translucidité à la surface de l'être ; et pour qu'il y ait conscience, il devra toujours y avoir « quelque chose » d'irréductiblement extérieur à elle.

\section{Face à l'autre : la liberté comme abandon (de la Raison)}

Jusqu'ici, nous avons examiné les rapports de la Raison et de la liberté dans un contexte spécifique : les relations entre la conscience et son monde. Mais

\footnotetext{
${ }^{1}$ Intentionnalité, p. 87.

${ }^{2}$ TI, p. 135 : « Le processus de la constitution qui se joue partout où il y a représentation, se renverse dans le "vivre de...". Ce dont je vis, n'est pas dans ma vie comme le représenté qui est intérieur à la représentation [...]. Si l'on pouvait encore parler ici de constitution, il faudrait dire que le constitué réduit à son sens, déborde ici son sens, il devient au sein de la constitution, la condition du constituant ou plus exactement la nourriture du constituant. Ce débordement de sens peut être fixé par le terme alimentation »).

${ }^{3}$ Cf. R. Burggraeve, " "Un roi déposé". Une voie de libération du moi par autrui », dans J. Hansel [dir.], Lévinas. De l'être à l'autre, Paris, PUF, 2006, p. 55-74.

${ }^{4} \mathrm{Et}$, donc, qui a besoin de quelque chose pour exister, à savoir, le « quelque chose » dont la conscience intentionnelle est toujours nécessairement conscience, pour la phénoménologie : l'étant.
} 
que deviennent ces rapports au sein des relations intersubjectives entre deux consciences ? Chez Lévinas, la rencontre de l'Autre renvoie dès le départ à la problématique d'une libération du sujet par rapport à l'être, considéré comme une « royauté indétrônable ${ }^{1}$. Cette libération est une évasion de l'être qui prend la forme d'une transcendance sans retour, car seule l'altérité de l'Autre peut libérer le moi de son enchaînement vis-à-vis du soi qui est constitué par l'activité intériorisante de la Raison. Face au visage de l'autre, cette activité doit être suspendue, car elle se configure en un impérialisme qui empêche toute possibilité d'éthique. L'évasion de l'être que prône Lévinas consiste donc en un véritable affranchissement du sujet par rapport à son existence et cette libération peut avoir lieu seulement grâce au rapport à une transcendance qui n'est pas assimilée à la conscience et qui, donc, implique une extase du moi sans qu'il y ait retour à soi. C'est donc l'Autre qui libère le moi de son enchaînement, en se configurant comme un objet sui generis, non-assimilable en raison de son caractère mystérieux ${ }^{2}$; comme objet d'un désir métaphysique d'infini ${ }^{3}$, dont le modèle est donné dans la troisième des Méditations Métaphysiques de Descartes ${ }^{4}$. Pour ce qui concerne le rapport de la conscience à la transcendance de l'Autre, Lévinas indique en effet ceci :

L'aspiration à l'extériorité radicale, appelée pour cette raison métaphysique, le respect de cette extériorité métaphysique qu'il faut, avant tout, " laisser être » - constitue la vérité. Elle anime ce travail et atteste sa fidélité à l'intellectualisme de la raison. Mais la pensée théorique, guidée par l'idéal de l'objectivité, n'épuise pas cette aspiration. Elle reste en deçà de ses ambitions. Si des relations éthiques doivent mener $[\ldots]$ la transcendance à son terme, c'est que l'essentiel de l'éthique est dans son intention transcendante et que toute intention transcendante n'a pas la structure noèse-noème ${ }^{5}$.

\footnotetext{
${ }^{1}$ E. Lévinas, Autrement qu'être ou au-delà de l'essence, Paris, Poche, 2016, p. 14. ${ }^{2}$ Ibid.

${ }^{3}$ Cf. TI, p. iv-44 ; Id., Hors l'expérience : l'idée cartésienne de l'infini, dans Dieu, la Mort et le Temps, Paris, Le Livre de Poche, 1995, p. 246-251 ; Id., De Dieu qui vient à l'idée, Paris, Vrin, 1982, p. 104-108 ; Id., La Philosophie et l'idée de l'infini, dans En découvrant l'existence avec Husserl et Heidegger, Paris, Vrin, 2016 (désormais cité $E E H H)$, p. 229-248.

${ }^{4}$ Sur cette question, $c f$. M. Dupuis, « Le cogito ébloui ou la noèse sans noème. Lévinas et Descartes », Revue Philosophique de Louvain n 94/2 (1996), p. 294-310 ; mais aussi J.-F. Lavigne, «L'idée de l'infini : Descartes dans la pensée d'Emmanuel Lévinas », Revue de métaphysique et de morale, ${ }^{\circ}$ 92/1 (1897), p. 54-66

${ }^{5}$ TI, p. 14-15.
} 
L'idée de l'infini est caractérisée par le fait de « déborder » ${ }^{1}$ la pensée qui la pense : dans la lecture lévinassienne, il n'y a donc aucune symétrie possible entre le moi et l'Autre ${ }^{2}$. Sur ce point se creuse non seulement la distance entre Lévinas et Sartre, concernant le rapport à autrui, mais c'est également ici qu'on voit poindre la critique du premier philosophe à l'encontre du second ${ }^{3}$ :

Autrui n'est pas transcendant parce qu'il serait libre comme moi. Sa liberté, au contraire, est une supériorité qui vient de sa transcendance même. En quoi consiste cette inversion de la critique ? Le sujet est « pour soi »-il se représente et se connaît aussi longtemps qu'il est. Mais en se connaissant ou en se représentant, il se possède, se domine, étend son identité à ce qui vient, en lui-même, réfuter son identité. Cet impérialisme du Même est toute l'essence de la liberté. Le « pour soi », comme mode de l'existence, indique un attachement à soi aussi radical qu'un vouloir naïf de vivre. Mais si la liberté me situe effrontément en face du non-moi, en moi et hors de moi, si elle consiste à le nier ou à le posséder, devant Autrui elle recule. Le rapport avec Autrui ne se mue pas, comme la connaissance, en jouissance et possession, en libertét .

Lévinas considère la liberté de l'Autre comme une transcendance, une hauteur, une supériorité par rapport au moi, dans un cadre non symétrique ni dialectique ; et il reproche à Sartre d'avoir pensé le rapport à l'Autre sur le modèle de la relation que la conscience entretient à l'égard de l'objet de la connaissance. Selon Lévinas, pour éviter la guerre face à l'Autre ${ }^{5}$, la liberté souveraine du moi doit reculer en abandonnant l'impérialisme qui conduit le sujet à vouloir posséder tout ce qui est « non-moi ».

Mais on sait que, chez Sartre, la structure intentionnelle de la conscience implique un écart entre les deux régions de l'être et pose derechef la liberté humaine en face d'un monde ontologiquement séparé, et inconnaissable de fa-

\footnotetext{
${ }^{1}$ Ibid., p. 10.

${ }^{2} T A$, p. 80 : « La relation entre maitre et esclave peut être saisie au niveau de la lutte, mais alors elle devient réciproque. Hegel a montré précisément comment le maître devient l'esclave de l'esclave et l'esclave le maitre du maître. En posant l'altérité d'autrui comme mystère défini lui-même par la pudeur, je ne la pose pas comme liberté identique à la mienne et aux prises avec la mienne, je ne pose pas un autre existant en face de moi, je pose l'altérité ».

${ }^{3}$ En ce qui concerne L'Être et le Néant, la critique qui est développée implicitement tout au long de Totalité et Infini. Sur ce point, voir G. Hanus, Échapper à la philosophie ? op. cit., p. 153-167.

${ }^{4}$ Ibid., p. 86.

${ }^{5}$ Cf. TI, p. 6.
} 
çon permanente puisqu'il est inappropriable. Donc, la conscience intentionnelle, qui est toujours conscience «de quelque chose», ne peut pas ne pas avoir d'objet. Dans une célèbre section de L'Être et le Néant, Sartre décrit, à travers l'expérience du regard, la rencontre de l'autre en terme de dialectique entre deux consciences libres et objectivantes qui ne peuvent rester simultanément telles qu'elles sont ; car la liberté de l'une fige en objet celle de l'autre, qu'elle rend « esclave $»^{1}$.

C'est la honte ou la fierté qui me révèlent le regard d'autrui et moi-même au bout de ce regard, qui me font vivre, non connaître la situation de regardé. Or, la honte [...] est honte de soi, elle est reconnaissance de ce que je suis bien cet objet qu'autrui regarde et juge. Je ne puis avoir honte que de ma liberté en tant qu'elle m'échappe pour devenir objet donné. Ainsi, originellement, le lien de ma conscience irréfléchie à mon ego-regardé est un lien non de connaître mais d'être. Je suis, par-delà toute connaissance que je puis avoir, ce moi qu'un autre connaît. Et ce moi que je suis, je le suis dans un monde qu'autrui m'a aliéné $[\ldots]^{2}$. Ainsi, être vu me constitue comme un être sans défense pour une liberté qui n'est pas ma liberté. C'est en ce sens que nous pouvons nous considérer comme des « esclaves », en tant que nous apparaissons à autrui ${ }^{3}$.

Et d'ajouter :

Ainsi autrui est d'abord pour moi l'être pour qui je suis objet, c'est-à-dire l'être par qui je gagne mon objectité. Si je dois seulement pouvoir concevoir une de mes propriétés sur le mode objectif, autrui m'est déjà donné. Et il est donné non comme être de mon univers, mais comme sujet pur. Ainsi ce sujet pur que je ne puis, par définition, connaître, c'est-à-dire poser comme objet, il est toujours là, hors de portée et sans distance lorsque j'essaie de me saisir comme objet. Et dans l'épreuve du regard, en m'éprouvant comme objectité non-révélée, j'éprouve directement et avec mon être l'insaisissable subjectivité d'autrui. Du même coup, j'éprouve son infinie liberté ${ }^{4}$.

La question du rapport à autrui relève ainsi, pour Lévinas et Sartre, de la liberté (et de l'infini). Mais la diversité de leurs perspectives renvoie à la possibilité pour une conscience de figer la liberté d'autrui en un objet (ou de subir cette dynamique). Là où Lévinas soutient que la conscience n'est pas intentionnelle face à l'altérité, Sartre refuse cette idée, même s'il lui arrive de reconnaître

${ }^{1}$ EN, p. 314.

${ }^{2}$ Ibid., p. 307.

${ }^{3}$ Ibid., p. 314.

${ }^{4}$ Ibid., p. 316-317. 
$l^{\prime}$ «absence ${ }^{1}$ essentielle de la conscience d'autrui. Lévinas semble en effet avoir retenu précisément ce point de la théorie sartrienne qui semble être le lieu où s'originent leurs différences théoriques fondamentales :

Et Sartre dira d'une façon remarquable, mais en arrêtant l'analyse trop tôt, qu'Autrui est un pur trou dans le monde. Il procède de l'absolument Absent. Mais sa relation avec l'absolument absent dont il vient, n'indique pas, ne révèle pas cet absent ; et pourtant l'Absent a une signification dans le visage. Mais cette signifiance n'est pas pour l'Absent une façon de se donner en creux dans la présence du visage - ce qui nous ramènerait encore à un mode de dévoilement. La relation qui va du visage à l'Absent, est en dehors de toute révélation et de toute dissimulation, une troisième voie exclue par ces contradictoires. [...] Mais [...] avons-nous été assez fidèles à l'interdiction de rechercher l'au-delà comme monde derrière notre monde ? L'ordre de l'être serait encore supposé ainsi [sic.]. Ordre qui ne comporte d'autre statut que celui du révélé et du dissimulé. Dans l'être, une transcendance révélée, s'invertit en immanence, l'extra-ordinaire s'insère dans un autre ordre, l'Autre s'absorbe dans le Même. [...] Le visage est précisément l'unique ouverture où la signifiance du Transcendant n'annule pas la transcendance pour la faire entrer dans un ordre immanent, mais où, au contraire, la transcendance se maintient comme transcendance toujours révolue du transcendant ${ }^{2}$.

Comme l'a noté Jean-Marc Mouillie, le projet sartrien « ne fut pas de fonder mais de démystifier pour libérer le caractère problématique de l'existence $»^{3}$. De même, dans un dialogue avec Philippe Némo, Lévinas déclare que sa « tâche ne consiste pas à construire l'éthique ; [mais] seulement à en chercher le sens $\gg{ }^{4}$; car, d'un point de vue pratique, la perspective lévinassienne ne pose pas moins de problèmes que celle sartrienne. Mais il n'était pas question ici de comparer les philosophies de Lévinas et de Sartre, en vue de dire laquelle était

${ }^{1}$ EN, p. 301-302 : « Mais autrui est encore objet pour moi. [...] [P]ar là la désintégration de mon univers est contenue dans les limites de cet univers même, il ne s'agit pas d'une fuite du monde vers le Néant ou hors de lui-même. Mais plutôt, il semble qu'il est percé d'un trou de vidange, au milieu de son être, et qu'il s'écoule perpétuellement par ce trou. L'univers, l'écoulement, et le trou de vidange, derechef tout est récupéré, ressaisi et figé en objet [...]. Tout cela, donc, ne nous fait nullement quitter le terrain où autrui est objet. Tout au plus, avons-nous affaire à un type d'objectivité particulier, assez voisin de celui que Husserl désigne par le mot d'absence ».

${ }^{2}$ E. Lévinas, La Trace de l'autre, dans EEHH, p. 276.

3 J.-M. Mouillie, « L'autodévoilement de la liberté dans l'existence (les horizons pratiques de l'ontologie de 1943) », dans R. Barbaras [dir.], Sartre. Désir et liberté, Paris, PUF, 2005, p. 192.

${ }^{4}$ E. Lévinas, Éthique et Infini, Paris, Fayard, 1982, p. 95. 
supérieure à l'autre, mais plutôt de montrer que leurs convergences et leurs divergences peuvent trouver à s'expliquer par leurs appropriations respectives du concept husserlien d'intentionnalité.

Bull. anal. phén. XVI 2 (2020)

https://popups.uliege.be/1782-2041/ @ 2020 ULiège BAP 